\title{
Relationships among Test Factor Structure, Test Hierarchical Structure, and Test Inter-Item Dependency Structure
}

\author{
William M. Bart and Rajkumari Palvia \\ University of Minnesota
}

In previous research, no relationship was found between test factor structure and test hierarchical structure. This study found some correspondence between test factor structure and test inter-item dependency structure, as measured by a log-linear model. There was an inconsistency, however, which warrants further study: More significant two-item interactions were found for the set of items that had been found to be most heterogeneous by latent trait techniques, rather than for the more homogeneous set of items.

The analysis of structure among dichotomously scored items in a test continues to be a central concern in psychometrics. The structure in a test has been conceived in two standard ways: (1) test factor structure, which is the complex of continuous latent traits (factors or dimensions) that the items assess and (2) test hierarchical structure, which is the system of prerequisite relations among the discrete items. Latent trait test theory has been used to investigate test factor structure (e.g., Bock \& Lieberman, 1970; Lord \& Novick, 1968), and ordering analysis has been used to investigate test hierarchical structure (Airasian \& Bart, 1973: Bart \& Airasian, 1974; Bart \& Krus, 1973).

Bart (1978) examined the relationship between these two types of test structure. He started with data for two sets of items such that one set of items

APPLIED PSYCHOLOGICAL MEASUREMENT

Vol. 8, No. 2, Spring 1984, pp. 199-205

(C) Copyright 1984 Applied Psychological Measurement Inc. 0146-6216/84/020199-07\$1.60 was established to have a unifactor structure and the other set of items a two-factor structure. These sets of item data were then analyzed with a refined ordering-analytic method to determine item hierarchies. No hierarchy structure was found for either set of items. Thus, Bart suggested that test factor structure provides little, if any, information about test hierarchical structure.

A third type of structure is the test inter-item dependency structure, which is the array of statistical interdependencies among the items. Discrete multivariate analysis, with its log-linear modeling, has been developed to investigate statistical interdependencies among discrete variables and can be used to investigate test inter-item dependency structure (Bishop, Fienberg, \& Holland, 1975). This study investigated the inter-item dependency structures of two tests whose test factor structures and test hierarchical structures have already been determined.

\section{Method}

\section{The Log-Linear Model}

Basic to the log-linear model is the cross-product ratio, $a$. Consider the case of two dichotomously scored items, $D_{1}$ and $D_{2}$, for which a $2 \times 2$ table can be constructed depicting four cell probabilities, $p_{00}, p_{01}, p_{10}$, and $p_{11}$, such that $p_{i j}(i=0,1$; $j=0,1)$ is the probability of receiving a score of 
$i$ for item $D_{1}$ and a score of $j$ for item $D_{2}$. A score of 1 indicates success on the item and a score of 0 indicates failure on the item. Using the $2 \times 2$ table in Table 1, the cross-product ratio can be defined as

$a=\frac{p_{00} \cdot p_{11}}{p_{10} \cdot p_{01}}$

The logarithm of the cross-product ratio, $a$, is a linear contrast of the log-probabilities of the four cells, namely,

$\log a=\log p_{00}-\log p_{10}$

$$
-\log p_{01}+\log p_{11} \text {. }
$$

When $\log a=0$, items $D_{1}$ and $D_{2}$ are said to be statistically independent. If $\log a>0$, items $D_{1}$ and $D_{2}$ are said to be statistically dependent.

To articulate certain elemental features of the log-linear model, the exposition provided by Bishop et al. (1975) will be closely followed. Proceeding in a manner analogous to analysis of variance (ANOVA) models, a linear model can be constructed with the use of the natural logarithms of the cell probabilities, e.g.,

$$
\begin{array}{r}
\log p_{u j}=u+u_{1(i)}+u_{2(j)}+u_{12(j)}, \\
i=0,1 ; j=0,1,
\end{array}
$$

where $u$ is the grand mean of the natural logarithms of the probabilities:

$$
\begin{aligned}
u=1 / 4\left(\log p_{00}\right. & +\log p_{01} \\
& \left.+\log p_{10}+\log p_{11}\right) .
\end{aligned}
$$

Then $u_{1(t)}$ is expressed as a function of the mean of the natural logarithms of the probabilities at level $i$ of the first item,

$$
\begin{aligned}
u_{1(i)} & =1 / 2\left(\log p_{i 0}+\log p_{i 1}\right)-u \\
i & =0,1,
\end{aligned}
$$

Table 1

An Inter-Item Contingency Table

\begin{tabular}{lll}
\hline & \multicolumn{2}{c}{$\mathrm{D}_{2}$} \\
\hline & 0 & 1 \\
\hline $\mathrm{D}_{1}$ & $\mathrm{P}_{00}$ & $\mathrm{P}_{01}$ \\
\hline 0 & $\mathrm{P}_{10}$ & $\mathrm{P}_{11}$ \\
\hline 1 &
\end{tabular}

and $u_{2(j)}$ is expressed as a function of the mean of the natural logarithms of the probabilities at level $j$ of the second item,

$u_{2(\jmath)}=1 / 2\left(\log p_{0 j}+\log p_{1 j}\right)-u$

$$
j=0,1 \text {. }
$$

With $u_{1(i)}$ and $u_{2(j)}$ representing deviations from the grand mean $u$,

$u_{1(0)}+u_{1(1)}=u_{2(0)}+u_{2(1)}=0$.

Also, $u_{12(i)}$ represents a deviation from $u+u_{1(i)}$ $+u_{2(j)}$; thus,

$u_{12(00)}=-u_{12(01)}=-u_{12(10)}=u_{12(11)}$.

If $t_{i j}=\log p_{i j}$, then the grand mean can be written as

$$
\begin{aligned}
u & =1 / 4\left(t_{++}\right) \\
& =1 / 4\left(t_{00}+t_{01}+t_{10}+t_{11}\right),
\end{aligned}
$$

with the main effects being

$u_{1(t)}=1 / 2\left(t_{t_{+}}\right)-1 / 4\left(t_{++}\right)$

and

$u_{2(j)}=1 / 2\left(t_{+j}\right)-1 / 4\left(t_{++}\right)$,

and the interaction term being

$u_{12(i j)}=t_{i j}-1 / 2\left(t_{i+}\right)-1 / 2\left(t_{+j}\right)$

$$
+1 / 4\left(t_{++}\right) \text {. }
$$

Bishop et al. (1975) closed their introductory section by pointing out that "the main effects are functions of the marginal sums of the logarithms but do not correspond to the marginal sums $p_{i+}$ and $p_{+j}$ in the original scale." Thus, item probability logarithms and their marginal sums become very important in this analysis.

The log-linear model for two items is analogous to a two-way ANOVA. Log-linear models for $n$ items, then, are analogous to $n$-way ANOVAs. This study investigated the interdependencies among two sets of five items and thus employed a loglinear model comparable to a five-way ANOVA.

\section{Test Data}

Either the items in each test data set are completely independent of each other or any two or more items are interdependent. The data for this study consisted of Items 11 through 15 of Section 6 (Figure Classification) and Section 7 (Debate) of the Law School Admission Test and was the same 
data analyzed by Bock and Lieberman (1970), Christoffersson (1975), and Bart (1978). To represent the two data sets, the responses of 1,000 subjects drawn from a larger sample of students applying for admission to law school at various universities in the United States were recorded as binary responses with " 1 " indicating a correct item response and " 0 " indicating an incorrect item re- sponse. Thus, there were $2^{5}=32$ possible item response patterns for each section of the test. The frequencies of the various item response patterns are recorded in Table 2 .

The data for both sets of items (LSAT6 and LSAT7) can be interpreted as constituting fivedimension contingency tables with each item representing a discrete variable and each item score

Table 2

Frequencies of Item Response Patterns

For Two Sets of Law School Admissions Test Items

\begin{tabular}{|c|c|c|c|c|c|c|c|}
\hline \multirow[b]{3}{*}{ Index } & \multirow{2}{*}{\multicolumn{5}{|c|}{$\begin{array}{c}\text { Response Pattern } \\
\text { of items } \\
\end{array}$}} & \multicolumn{2}{|c|}{ Frequency } \\
\hline & & & & & & \multirow{2}{*}{$\begin{array}{l}\text { LSAT6 Figure } \\
\text { Classification }\end{array}$} & \multirow{2}{*}{$\begin{array}{l}\text { LSAT7 } \\
\text { Debate }\end{array}$} \\
\hline & 1 & 2 & 3 & 4 & 5 & & \\
\hline 1 & 0 & 0 & 0 & 0 & 0 & 3 & 12 \\
\hline 2 & 1 & 0 & 0 & 0 & 0 & 10 & 7 \\
\hline 3 & 0 & 1 & 0 & 0 & 0 & 1 & 10 \\
\hline 4 & 1 & 1 & 0 & 0 & 0 & 16 & 6 \\
\hline 5 & 0 & 0 & 1 & 0 & 0 & 1 & 3 \\
\hline 6 & 1 & 0 & 1 & 0 & 0 & 3 & 14 \\
\hline 7 & 0 & 1 & 1 & 0 & 0 & 0 & 7 \\
\hline 8 & 1 & 1 & 1 & 0 & 0 & 11 & 18 \\
\hline 9 & 0 & 0 & 0 & 1 & 0 & 2 & 1 \\
\hline 10 & 1 & 0 & 0 & 1 & 0 & 14 & 11 \\
\hline 11 & 0 & 1 & 0 & 1 & 0 & 0 & 3 \\
\hline 12 & 1 & 1 & 0 & 1 & 0 & 21 & 7 \\
\hline 13 & 0 & 0 & 1 & 1 & 0 & 3 & 3 \\
\hline 14 & 1 & 0 & 1 & 1 & 0 & 15 & 15 \\
\hline 15 & 0 & 1 & 1 & 1 & 0 & 2 & 8 \\
\hline 16 & 1 & 1 & 1 & 1 & 0 & 28 & 32 \\
\hline 17 & 0 & 0 & 0 & 0 & 1 & 6 & 19 \\
\hline 18 & 1 & 0 & 0 & 0 & 1 & 29 & 39 \\
\hline 19 & 0 & 1 & 0 & 0 & 1 & 8 & 5 \\
\hline 20 & 1 & 1 & 0 & 0 & 1 & 56 & 25 \\
\hline 21 & 0 & 0 & 1 & 0 & 1 & 1 & 19 \\
\hline 22 & 1 & 0 & 1 & 0 & 1 & 28 & 51 \\
\hline 23 & 0 & 1 & 1 & 0 & 1 & 3 & 23 \\
\hline 24 & 1 & 1 & 1 & 0 & 1 & 61 & 136 \\
\hline 25 & 0 & 0 & 0 & 1 & 1 & 11 & 7 \\
\hline 26 & 1 & 0 & 0 & 1 & 1 & 81 & 34 \\
\hline 27 & 0 & 1 & 0 & 1 & 1 & 16 & 7 \\
\hline 28 & 1 & 1 & 0 & 1 & 1 & 173 & 35 \\
\hline 29 & 0 & 0 & 1 & 1 & 1 & 4 & 17 \\
\hline 30 & 1 & 0 & 1 & 1 & 1 & 80 & 90 \\
\hline 31 & 0 & 1 & 1 & 1 & 1 & 15 & 28 \\
\hline 32 & 1 & 1 & 1 & 1 & 1 & 298 & 308 \\
\hline
\end{tabular}


as a variable category. Since the responses are binary for each item, there are only two categories for each item variable, thus composing a $2 \times 2$ $\times 2 \times 2 \times 2$ contingency table.

Let $\log p_{i j k l m}$ denote the logarithm of the probability of the $(i, j, k, l, m)$ cell in a five-way contingency cell. Then, a "saturated"' log-linear model for the data can be written as

$$
\begin{aligned}
& \log p_{i j k l m}= \\
& u+u_{1(i)}+u_{2(\jmath)}+u_{3(k)}+u_{4(l)}+u_{5(m)} \\
& \quad+u_{12(i j)}+u_{13(l k)}+\ldots+u_{35(\mathrm{~km})}+u_{45(l m)} \\
& +u_{123(i j k)}+\ldots+u_{345(\mathrm{klm})} \\
& \quad+u_{1234(i j k l)}+\ldots+u_{2345(\mathrm{jklm})} \\
& +u_{12345(i j k l m)}
\end{aligned}
$$$$
\text { with } i=0,1 ; j=0,1 ; k=0,1 ; l=0,1 ; m=
$$$$
0,1 \text {. }
$$

The $u$-terms $u_{1}, u_{2}, u_{3}, \ldots, u_{12345}$ are parameters of the model and have the usual meaning as in a general log-linear model. (For more details, consult Bishop et al., 1975.) The number of independent parameters in the saturated model being used is 32 , which is the number of cells in the five-way contingency table representing each data set.

\section{Results}

Models derived from the five-variable saturated model were used in this analysis. Both the chisquare statistic, $\chi^{2}$, and the likelihood ratio statistic, $G^{2}$, were calculated for each model. Only hierarchical models were fitted to the data, which means that if any of the $u$-terms is set to zero, all of its higher-order relatives must also be zero. For example, if $u_{12}=0$, then $u_{123}, u_{124}, u_{125}, u_{1234}, u_{1235}$, $u_{1245}$, and $u_{12345}$ must also be zero (Bishop et al., 1975).

The first model examined was the model of independence, which can be represented as

$$
\begin{aligned}
\log p_{i j k l m}=u & +u_{1(i)}+u_{2(j)} \\
& +u_{3(k)}+u_{4(l)}+u_{5(m)} .
\end{aligned}
$$

This model was fitted to the two sets of data, and the $\chi^{2}$ values for both sets were quite large. The $\chi^{2}$ value for the LSAT6 (Figure Classification) data was 95.06 with $p<.005$ and the $\chi^{2}$ for the LSAT7 (Debate) data was 356.6 with $p<.005$. Hence, the hypothesis of independence among the test items was rejected for both sets of items, suggesting that one or more interaction terms were present within both the LSAT6 the LSAT7 item sets.

The next step in the analysis was to find the interaction(s) present in each set of data (i.e., the interactions that were significantly different from zero). The model with all two-factor interactions present, but all higher-order interaction terms being zero, fit very well each of the two data sets. This result is quite important in that it established that (1) there were no complex three- or four- or fivefactor interactions present among the items of both sets of data and that (2) the presence of significant second-order interactions could be compared directly with the inter-item correlations presented by Bock and Lieberman (1970). This comparison is discussed at greater length below.

The next step was to see which of the two-factor interaction terms could be further eliminated from the model of all two-factor interactions (with no higher-order interactions being present) without significantly lowering the $p$-value.

The 10 models with 1 two-factor interaction term being removed for each model were fitted to each set of test item data. Tables 3 and 4 present the most prominent results of these analyses for the LSAT6 and LSAT7 data sets, respectively. For LSAT6 data, the $u_{15}=0$ model fit the best of all 10 models without even affecting the $p$-value. Eliminating $u_{15}$, there were only 9 two-factor interaction terms in the model. As was done previously, 9 models were fitted with one interaction term (in addition to $u_{15}$ ) being removed at a time. The term $u_{14}$ was next to be eliminated from the original model. The procedure was repeated until no more interaction terms could be removed. This same procedure was repeated for LSAT7 data.

The selection of the best model depends on the $p$-value set by the experimenter or by the purpose of the study. If through the elimination of an interaction term, there is a sudden drop in $p$-value, then it may be desirable to maintain that interaction term in the model. Using that principal in the fitting of the various interaction models, the best model for the LSAT6 data was found to be 
Table 3

The Fit of Each of Several Log-linear Models

for a Set of Five Law School Admissions Test (LSAT6) Items

Assessing Figure Classification Ability

Mode1

$\mathrm{G}^{2} \quad \chi^{2}$

$\mathrm{df}$

$\mathrm{p}$

(1) Independence with all

interactions absent

(2) All three factor and higher-order interactions absent

(3) $\mathrm{u}_{15}=0$

(4) $u_{15}=u_{14}=0$

(5) $u_{15}=u_{14}=u_{35}=0$

(6) $u_{15}=u_{14}=u_{35}=u_{24}=0$

(7) $\mathrm{u}_{15}=\mathrm{u}_{14}=\mathrm{u}_{35}=\mathrm{u}_{24}=\mathrm{u}_{12}=0$

(8) $\mathrm{u}_{12}=\mathrm{u}_{14}=\mathrm{u}_{15}=\mathrm{u}_{24}=\mathrm{u}_{25}=$ $\mathrm{u}_{35}=0$

(9) $\mathrm{u}_{12}=\mathrm{u}_{14}=\mathrm{u}_{15}=\mathrm{u}_{24}=\mathrm{u}_{25}=$ $\mathrm{u}_{34}=\mathrm{u}_{35}=0$

(10) $\mathrm{u}_{12}=\mathrm{u}_{14}=\mathrm{u}_{15}=\mathrm{u}_{23}=\mathrm{u}_{24}=$ $\mathrm{u}_{25}=\mathrm{u}_{34}=\mathrm{u}_{35}=0$

\subsection{0}

95.06

26

$<.005$

16.48

14.65

16

$>.25$

16.59

15.34

17

$>.25$

17.45

16.46

18

$>.25$

18.62

17.11

19

$>.25$

20.36

19.38

20

$>.25$

24.06

24.95

21

ح.25

30.93

34.96

22

$=.1$

42.78

47.74

23

$<.001$

236.1

229.0

24

$<.005$ $\log p_{i j k l m}=u+u_{1(i)}+u_{2(j)}+u_{3(k)}$

$$
\begin{aligned}
& +u_{4(l)}+u_{5(m)}+u_{13(i k)} \\
& +u_{23(j k)}+u_{25(j m)}+u_{34(k l)} \\
& +u_{45(l m)}
\end{aligned}
$$

This model has only 11 independent parameters as compared to the original model. The best model describing the LSAT7 data was found to be

$$
\begin{aligned}
\log p_{u k l m}=u & +u_{1(t)}+u_{2(j)}+u_{3(k)} \\
& +u_{4(l)}+u_{5(m)}+u_{12(j)} \\
& +u_{13(i k)}+u_{14(i l)}+u_{15(i m)} \\
& +u_{23(j k)}+u_{24(j)}+u_{34(k l)} \\
& +u_{35(k m)},
\end{aligned}
$$

which has 14 independent parameters.

For the LSAT6 data, the only interaction term present in the $u_{12}=u_{14}=u_{15}=0$ model relating to Item 1 was $u_{13}$. This implies that Item 1 was related only to Item 3 and is conditionally independent of all of the other items. Since $u_{13}, u_{23}$, and $u_{34}$ were present in the model, but $u_{35}=0$, Item 3 was found to be related to all of the items except Item 5.

For the LSAT7 data, all interaction terms containing Items 1 and 3 -such as $u_{12}, u_{13}, u_{14}, u_{15}$, $u_{23}, u_{34}$, and $u_{35}$-were significant and thus present. This condition implied that Item 1 was related to all of the other items, and the same was the case with Item 3 . Since $u_{25}=u_{45}=0$, Item 2 was related to all of the other items, and the same was the case with Item 3 . Since $u_{25}=u_{45}=0$, Item 2 was related to all of the other items except Item 5 . Item 4 was also related to all of the other items except Item 5.

The results of this study seem to be in accordance 


\author{
Table 4 \\ The Fit of Each of Several Log-linear Models \\ for a Set of Five Law School Admissions Test (LSAT7) Items \\ Assessing Debate Ability
}

\begin{tabular}{|c|c|c|c|c|}
\hline Mode1 & $G^{2}$ & $x^{2}$ & df & $\mathrm{p}$ \\
\hline $\begin{array}{l}\text { (1) Independence with a11 } \\
\text { interactions absent }\end{array}$ & 200.9 & 356.6 & 26 & $<<.005$ \\
\hline $\begin{array}{l}\text { (2) AlI three factor and } \\
\text { higher order inter- } \\
\text { actions absent }\end{array}$ & 20.38 & 20.14 & 16 & $.2<\mathrm{p}<.25$ \\
\hline (3) $\mathrm{u}_{25}=0$ & 20.86 & 20.85 & 17 & $.2<p<.25$ \\
\hline (4) $u_{45}=u_{25}=0$ & 23.02 & 23.50 & 18 & $.1<\mathrm{p}<.2$ \\
\hline (5) $\mathrm{u}_{12}=\mathrm{u}_{45}=\mathrm{u}_{25}=0$ & 28.40 & 28.54 & 19 & $.05<\mathrm{p}<.1$ \\
\hline (6) $\mathrm{u}_{24}=\mathrm{u}_{45}=\mathrm{u}_{25}=0$ & 28.82 & 29.78 & 19 & $.05<p<.1$ \\
\hline (7) $\mathrm{u}_{24}=\mathrm{u}_{12}=\mathrm{u}_{45}=\mathrm{u}_{35}=0$ & 36.05 & 36.61 & 20 & $.01<p<.05$ \\
\hline
\end{tabular}

with the results regarding the tetrachoric correlation coefficients reported by Bock and Lieberman (1970). For the LSAT6 data, the interaction terms of $u_{14}$, $u_{15}, u_{24}$, and $u_{35}$ were the first four interaction terms to be eliminated from the model of all two-factor interactions; this situation was reflected in the correlation coefficients for item pairs $(1,4),(1,5),(2,4)$, and $(3,5)$ being the smallest inter-item correlations cited for the LSAT6 data by Bock and Lieberman (1970). Similarly, the item pairs $(2,5)$ and $(4,5)$ engendered the two smallest inter-item correlations cited for the LSAT7 data by Bock and Lieberman.

One result emanating from this study that conflicted with results from the studies by Bock and Lieberman (1970) and by Christoffersson (1975) concerned the heterogeneity of the items. These studies contended that the LSAT7 items were more heterogeneous than the LSAT6 items, but there were larger inter-item tetrachoric correlation coefficients among the LSAT7 items, and there were more significant interaction terms present in the best-fitting log-linear model for the LSAT7 data than in the best-fitting model for the LSAT6 data. Despite the results of the latent trait test theoretic analyses performed on the data in those two stud- ies, the LSAT7 items may have been much less heterogeneous than reported. A closer examination of the similarities and differences among the items is needed to identify item features that may contribute to the homogeneity among items.

\section{Discussion}

Bart (1978) established that two sets of test itemsone with a unifactor structure and the other with a two-factor structure - had (1) no hierarchical structures, (2) a prominent first linear order but no clear uniorder or two-order structures, and (3) internal inconsistency. He determined empirically that factor structures and hierarchical structures cannot be simply mapped into each other. In this study, an array of significant two-item interactions were located for the two test item sets, with more significant two-item interactions being located for the set of items found to be most heterogeneous by latent trait techniques. To be more specific, the five LSAT6 items were determined to be more interdependent by a log-linear analysis and more homogeneous by latent trait techniques than the five LSAT7 items. These results could very likely be reflected in the 
complete sections of Figure Classification and Debate items, respectively, in the LSAT. This apparent inconsistency warrants further examination before an adequate explanation can be generated. It would be assumed that there would be substantial correspondence between the test factor structure and the test inter-item dependency structure, but the results of this study indicated that the correspondence is not a strong one. It is suggested that all three types of test structure be investigated for any test item data set and that the applied consequences of each of the three structures be investigated.

\section{References}

Airasian, P., \& Bart, W. (1973). Ordering theory: A new and useful measurement model. Educational Technology, 13, 56-60.

Bart, W. (1978). An empirical inquiry into the relationship between test factor structure and test hierarchical structure. Applied Psychological Measurement, 2, 333337.

Bart, W., \& Airasian, P. (1974). The determination of the ordering among seven Piagetian tasks by an ordering-theoretic method. Journal of Educational Psychology, 9, 251-259.

Bart, W., \& Krus, D. (1973). An ordering-theoretic method to determine hierarchies among items. $E d u$ - cational and Psychological Measurement, 33, 291300.

Bishop, Y., Fienberg, S., \& Holland, P. (1975). Discrete multivariate analysis: Theory and practice. Cambridge MA: MIT Press.

Bock, R., \& Lieberman, M. (1970). Fitting a response model for $n$ dichotomously scored items. Psychometrika, 35, 179-197.

Christoffersson, A. (1975). Factor analysis of dichotomized variables. Psychometrika, 40, 5-32.

Lord, F., \& Novick, M. (1968). Statistical theories of mental test scores. Reading MA: Addison-Wesley.

\section{Acknowledgments}

The research reported herein and the preparation of this paper were supported in part by grants to the authors from the United States Army Research Institute for the Behavioral and Social Sciences. Preparation of this paper was also supported in part by grants to the University of Minnesota, Center for Research in Human Learning, from the National Science Foundation (Grant No. GB$35703 \mathrm{X}$ ), from the National Institute of Child Health and Human Development (Grant No. HD-00136), and from the Graduate School of the University of Minnesota.

\section{Author's Address}

Send requests for reprints of further information to William M. Bart, 330 Burton Hall, University of Minnesota, 178 Pillsbury Drive SE, Minneapolis MN 55455, U.S.A. 